load in her stomach after meals. Organs generally found healthy exceptstomach, which was moderately dilated, extend ing down to half an inch below the umbilicus, with delayed emptying. The gastric analysis showed only a very small amount of free $\mathrm{HCl}(.014)$ though the T. A. was 66 , representing largely fermentation products. There was much mucus in the stomach. I advised lavage and the combination of $\mathrm{HCl}$ and pepsin as the main treatment. On account of marked starch indigestion, she also took 'Taka-Diastase for a time, and Roncegno Water was taken for some weeks to bring up the quality of the blood.

On April 9, 1898, I found the stomach much retracted in size, the lower border being one and a half inches above the um bilicus, and the gastric analysis showed T. A. 56 ; free $\mathrm{HCl}$ 075; mucus a very small amount. She had had no severe headaches with vomiting for three months, and had regained a normal color, though her gastric catarrh is not yet entirely well.

Case 4-Gentleman, aged 66, consulted me July 21, 1897, in A tlantic City on account of chronic indigestion, from which he claimed to have suffered nearly all his life. Painful accumulations of gas and obstinate constipation were prominent features. The external physical examination revealed nothing abnormal. Gastric analysis: T. A. 16 ; free $\mathrm{HCl}$ wanting. Starch digestion good. Mucus very small amount. He was placed upon $\mathrm{HCl}$ and pepsin in the usual way. He has since seen me several times on account of his wife, but reported that he himself was doing so well on the digestive mixture as not to require any further medical assistance.

December 16, he was seen and was then feeling well. On A pril 22, 1898, his wife called to consult $m \theta$ for herself and re ported that her husband had been continuing his $\mathrm{HCl}$ and pepsin, though less regularly, having virtually recovered his health. He had taken no other medicine except a little nux vomica during the first few weeks and a laxative at night. He had not had massage except such kneading over the abdomen as he had been able to give himself.

In this case no opportunity has been offered of testing the stomach contents again, but it is highly probable, from the decided improvement in the patient's digestion as well as in his general health, that the gastric glands are now doing much better work

Reports of a number of other cases might be added in which under a treatment consisting either entirely or mainly of the administration of $\mathrm{HCl}$ and pepsin, conditions of apepsia or hypopepsia improved more or less markedly, the gastric secre tion having returned to the normal. Without claiming that such fragments of clinical experience can be accepted as demonstrating beyond question that $\mathrm{HCl}$ stimulates the gastric glands, it must be admitted that a strong presumption is thus established as to the existence of such an action. REFERENCES,

1 Die Erkrankungen des Magens. Vienna, 1896

2 Diet in the Chronic Catarrhs of the Gastro-Intestinal Tract. Jour Amer. Med. Ass., Feb. 19, 1898; and Important Indications and Contrandications for Massage of the Abdomen. Inter. Med. Mag., Jan., 1898.

Diseases of the Stomach. By Dr. John W. Hemmeter, Phla. 1897. Nisbet. Phila. 1898.

5 Therapie derVerdauungskrankheiten. Von Dr.CarlWegele, Jan., 1895.
6 Loc. citat.

\section{RECENT THERAPEUTIC APPLICATION OF THE VALERIANATES OF CREO- SOTE AND GUAIACOL.}

Presented to the Section on Materia Medica, Therapeutics and Pha macy, at the Forty-ninth Annual Meeting of the American Medical Association, held at Denver, Colo., June 7-10, 1898.

BY J. W. WAINWRIGHT, M.D.

NEW YORI CITY.

It can be safely assumed that the marvelous evidences of progress which the world has witnessed during the last quarter of a century in the science of medicine, especially in the department of clinical medicine and surgery, in very great measure muy be justly attributed to the discovery of the relationship which exists either directly or indirectly between certain diseases and a comparatively small group of micro-organisms which are now known to be pathogenic. Out of this application of bacteriology or microscopic botany to the practice of medicine has been developed our present aseptic and antiseptic methods of wound treatment and also our therapeutics of many infectious diseases. The knowledge that we now have of the causes of infection in wounds we owe to the genius of Lister, who has taught us also how we may securely guard against suppuration and other germinduced pathologic conditions. Similarly Pasteur, Koch, Klebs and others have not only enlarged our sphere of information as to the nature of many diseases to which mankind is liable, but have also clearly indicated the only means of preventing their future progress by isolation and disinfection.

Sepsis therefore occupies an important position in the mind of the clinician, and in consequence, imperative demand has arisen for a class of remedies known as antiseptic agents on account of their ability to counteract the pernicious activity of pathogenic microorganisms. The chemists have been diligent in their investigations and have placed in the hands of the therapeutist powerful weapons to be used in the warfare between the human body and the invading hosts of disease-breeding cocci and bacteria.

We must not fail to recognize and fully acknowledge the important rôle played by the white blood corpuscles in protecting the human organism from infection; without such natural antiseptics the human race would have disappeared many centuries ago. The aim of therapeutics in this class of diseases is to aid in the battle between the leucocytes and the germs by increasing the functional activity of the former and weakening the virulency of the latter. It is also im. portant to acknowledge the indispensable aid to be derived from hygienic measures in this direction, and especially the great value of sunlight, pure air, and wholesome, easily assimilated food, all of which, from this standpoint, may be regarded as accessory antiseptic agents.

Many of the chemic antiseptics are poisonous to all forms of life, and when these agents are used in medicine it is necessary that we be careful that in our efforts to destroy the bacteria, we do not inflict permanent injury to the patient. One of the most remarkable of our antiseptic remedies and at the same time perhaps, the least poisonous to the human system, is beechwood creosote, which has had an estab. lished place in medicine for more than half a century; but as you are aware, creosote has been largely adulterated in the shops and impure carbolic acid substituted, and thus the best results were not at first secured. As a result of Sommerbrodt's efforts on behalf of creosote from beechwood, however, it received greater consideration in the treatment of phthisis, and this interest resulted in our obtaining it finally in a pure state. Creosote being a compound of various elements, chiefly guaiacol and creosol, it was thought by some that more favorable results might be obtained from the principal ingredient, guaiacol, and this was isolated and used with results known to all. In these remedies we found prompt antiseptic and germicidal properties with less poisonous effects upon human beings than from the common phenol or carbolic acid, which in the early day of Listerism was the most prominent among our antiseptic agents. Guaiacol has also become widely known through its remarkable power of reducing temperature when applied to the surface of the body, which discovery is due to Sciolla.

This brings us to the consideration of the newer of the many derivations or compounds of creosote, the valerianates of creosote and guaiacol, for brevity denominated eosot and geosot. They are nearly neutral 
esters, having an odor resembiing somewhat that of $/$ stituents, guaiacol or creasote and valerianic acid. the English walnut, and boiling under normal atmos. This would be the usual method, but it might occur pheric pressure at about 260 degrees $C$; are soluble in that the alkalinity of the intestinal secretions would alcohol, ether and benzine. They are administered not be of sufficient degree to decompose a compound, internally in coated gelatin capsules, each containing especially if the remedy were of a character to resist $3 !$ min. and in liquid form either diluted with abso- its action. This would occur to a greater or less lute alcohol and flavored with oil of peppermint, cin- extent with a combination whose constituents were namon or cloves or directly in milk. The dose is chemically firmly fixed. The more stable the combifrom $33_{3}^{1}$ to 9 min. three times a day.

A convenient formula is as follows:

B. Eosot or geosot, $20 \mathrm{ccm}$. (fl. dr. v).

Aleohol 95 per cent., $80 \mathrm{ccm}$. (fl. oz. ii dr. v).

Ol. menth. pip., $0.6 \mathrm{ccm}$. (gtt. $\mathrm{x}$ ).

M. Each tablespoonful contains about $12 \mathrm{~min}$. of eosot or geosot. Mix dr. i to dr. iii with plenty of milk and give this dose in three equal parts daily.

It is generally conceded that in the treatment of phthisis, for a decade at least, creosote or its derivatives have given the best results of any of the multitude of remedies lauded, but creosote has been so pr destructive to the digestive and alimentary functions that but seldom a patient would be found who could continue its use sufficiently long or in large enough doses to produce a lasting or permanent effect. The poor patient would lose his appetite and desire for food and consequently his physical strength and power to battle with the disease. Besides this, there was ever present the depressing and self-conscious factor of his own limitutions toward ultimate recovery increased by the never-ceasing gastric distress.

The valerianates seem to meet all the requirements as remedies destructive to this disease without unto. ward effects. They increase the appetite and weight, relieve cough, hectic and night sweats. The valerianic acid component, by its sedative action on the nerve centers, particularly the sympathetic, enables the patient to enjoy quiet and refreshing sleep as well as a sense of euse and comfort not secured by other remedies. Being eliminated largely by the mucous membrane their bacteriologic effects are quickly apparent when used internally in bronchitis, acute gustritis, chronic gastrectasia, typhoid fover, and intestinal irritation; when used externally in lupus and all purulent inflammations, as well as subcutaneously in glandular enlargements of tubercular origin, in bip joint and other bone complications, and as a spray in all nose and throat affections.

Physiologic action-Providing the amount of acidity of the secretions be sufficient to replace that of the acid in the combinations, the valerianates of creosote and guaiacol would be decomposed in the stomach. The hydrochloric acid of the gastric secretion varies greatly in different people as well as in the same patient from day to day and as the estimate is made before or after meals. There is also a great variation of acidity in consequence of the character of the diet as well as at different periods of the day. All these conditions must be considered in determining to what extent a combination will be separated into its several constituents in the stomach; hydrochloric, being the stronger of the acids, would under certain conditions replace the valerianic acid or have the effect of decomposing the combination of the valerinate, thus releasing the creosote or guaiacol in the stomach, but such a degree of acidity would very rarely occur, and without an abnormally large amount of hydrochloric acid one would not find the decomposition take place there, but that the remedy would pass on into the intestines and upon encountering the acid antagonist, alkalinity, would then and there be resolved into the two connation the more surely would a greater or less amount escape in the feces unchanged. It is not at all probable that this decomposition is brought about by the ferments or the products of fermentation, as has been claimed necessary for the decomposition of the carbonates of creosote and guaiacol, but that the remedies would act largely in the alimentary tract as they do in the test tube, due allowance being made for slight variations not likely to be material. If the liberation of the several components depended upon the products of fermentation alone, or even to a quite considerable extent, we would not be able to secure the germicidal effects of the creosote or guaiacol in organs or tissue remote from the iutestinal tract as in phthisis, for the creosote or guaiacol having once rendered the intestinal tract aseptic it would remain so for a considerable time, and if the remedy were continued it must necessarily pass on through unchanged, not encountering the products of fermentation necessary to decompose it.

This theory can not be reasonably assumed, for clinically we know that the creosote or guaiacol can be pushed to such an extent as to completely saturate the organism. It has been claimed that the extreme volatility of the valerianates admits of their absorption directly into the circulation as the valerianates, and without the necessity of a chemic action; that they are separated into their component parts after entering the circulation by the alkalin constituents of the blood, as is evidenced by the odor of creosote or guaiacol so quickly appearing upon the handkerchiefs of those using them for coryza. This property is of great advantage, as it is apparent that the patient would thus be quickly under their influence without the necessity of chemic changes in the intestinal tract to prepare them for absorption by a decomposition necessary in the more stable compounds such as salol, resorcin, the carbonates, etc. To this property may be attributed the small doses necessary when compared with other remedies, as the whole of the valerianates is utilized.

As regards the rationale of the action of guaiacol, ${ }^{1}$ it has been found that "the beneficial effect of guaiacol in tuberculosis is chiefly due, not to any direct action upon the digestive organs or the blood, but to its combination in the blood with the albuminous bodies (toxalbumins) derived from the growth of the bacilli; that the absorbed guaiacol combines with these products and renders them barmless, and that they are further changed by oxidation, the guaiacol being liberated as a salt of ethyl-sulphuric acid, and the other decomposition products being eliminated in the urine. The products of the bacilli bring about the fever sweating, disordered digestion, etc., and with their destruction the ill effects pass away." This appears a very probable explanation and it is evident that an organic compound such as the ester of valerianic acid, which is quickly and easily decomposed, yielding nascent guaiacol in a condition ready for immediate absorption into the blood-vessels, is the most efficient

1 Berliner Klinische Wochenschrift, Jan. 18, 1892. 
and economical method of administering this invalu- according to the experience had with the eosot in the able remedy. It would also seem that this is the best Gerhardt Clinic, we can say that it fulfils all the form in which to give guaiacol in the treatment of requirements of a reliable preparation which can be typhoid fever and other intestinal disorders.

'The clinical results obtained with the valerianates of creosote and guaiacol may be summarized as follows:

Dr. Rieck, Bassum, Germany, has an article in the August number of the Medizinal Zeitung of Berlin, August, 1897, on the valerianate of guaiacol in the treatment of tuberculosis which shows the following results: Of seventy-six cases treated in the various stages and forms of tuberculosis, he reports thirty-one cures, and thirty-two of the remainder so much improved as to have almost reached complete recovery; nineteen of the thirty-two giving up all treatment because they felt perfectly well. There were eight fatal cases in the whole number treated; rapid and extensive destruction of the lung three; cerebral tuberculosis two, and general tuberculosis three. The Doctor says that during his whole experience he has never been able to obtain results which even approximated those treated with the valerianate of guaiacol (geosot), not only in tuberculosis but in bronchitis, especially of children, in broncho-pneumonia, catarrh of the bronchi, larynx, pharynx and nasal passages; in acute gastric catarrh and in bone tuberculosis by injection directly into the bone tissue, and finally in lupus. In the cases of catarrh of the apex treated, twenty-four in all, there was quick and permanent relief, night sweats disappearød, sensitiveness to atmospheric changes lessened, weight and appetite increased and the cough was reduced to an occasional evacuation of mucus which soon became less tenacious and was eas. ily ejected. It acts pre-eminently upon the mucous membranes and is therefore useful in diseases of the stomach, intestines and the respiratory passages. It is especially a remedy for tuberculosis, producing no irritation whatever, but in a fow days bringing a feeling of comfort and ease.

Dr. W. Zinn of the "Second Medical Clinic Uni. versity of Berlin," known as Professor Gerhardt's Clinic, published in Therapentische Monatschefte, Berlin, 1898, a communication entitled "Further Results with the Valerianate of Creosote (eosot)," in which he states that: "Dr. Grawitz reported in the Therapeutische Monatschefte, Berlin, July, 1896, thirty-five cases of tuberculosis treated with the ralerianate of creosote (eosot) as well as a number of stomach and intestinal disturbances, stating that the remedy was taken in all cases without ill effects to the digestive functions. I have now to report that since the publication of Dr. Grawitz's article we have treated eighty more cases of tuberculosis with the valerianate of creosote. As a rule, the remedy was given in capsules, each containing $3 \frac{1}{3} \mathrm{~min}$., one or more capsules three times a day. In several cases the medicament was continued for several months. It was used in all forms of tuberculosis and it was especially noted that there was in no case disturbance of the gastro-intestinal canal. It is accepted that of all the many remedies which have been recommended for tuberculosis the beech-wood creosote alone retains a prominent ṕlace notwithstanding its use has so often been productive of disagreeable effects. The valerianate of creosote is therefore worthy of especial mention because of its freedom from these disturbing influences. Particularly in the early stages of tuberculosis bert, the physician in charge, in an article published the eosot will be found of great service. Finally, in the New York Medical Journal, April 2, 1898, 
says that "geosot, valerianate of guaiacol, proved an agreeable form of administering guaiacol. It was found less irritating to the stomach than creosote." I have very little time to refer to other valuable uses of eosot and geosot, such as their value in overcoming irritability of the stomach as in cases reported by Dr. W. E. Anthony of Providence, R. I., where in the nausea of early pregnancy and the sick stomach of Bright's disease of the kidneys they gave prompt relief. Having indicated some of the applications of the valerianates of creosote and guaiacol in clinical medicine and surgery, with one further reference I will close. Surgically Dr. Rieck refers to two cases of lupus of the face cured by applications of geosot and also to two cases of tuberculosis or white swelling of the knee and hip joint cured by injections of geosot into the capsule of the joint. Dr. Rieck also positively states that the valerianate of guaiacol cures bone tuberculosis, though not through the usual channels of absorption but by direct local application and declares that there is no question but that the use of the valerianate of guaiacol will permit of a marked extension of conservative measures in the treatment of tuberculosis of the bones and joints.

\section{THE GENERAL PRINCIPLES OF ALKA- LOIDAL MEDICATION.}

Presented to the Section on Materia Medica, Pharmacy and Therapeutics at the Forty-ninth Annual Meeting of the American Medical Association, held at Denver, Colo., June 7-10, 1898. BY W. C. ABBOT'T, M.D.

CHICAGo.

My purpose in coming before you is to present some of the general principles governing that form of medicinal treatment which I have denominated "Alkaloidal Medication," and I trust that I may be enabled to present them with sufficient force and clearness to make my meaning distinctly understood. I am prompted to this effort by the belief that by bringing the subject before this Section of the great Association, we may correct some mistakes which my experience shows to exist very generally among the members of our profession.

There is an erroneous idea abroad that those who favor alkaloidal or dosimetric medication aim to establish a distinct system of practice. This is a great mistake, and a careful perusal of the literature of the subject will show that the word "system" is used by its opponents while the word "method" is used by its friends. In other words, what we claim for alkaloidal medication is that it is simply an up-todate method for the treatment of disease, whereby departures from physiologic equilibrium can be controlled more quickly, safely and pleasantly than by any other known method. This claim we base upon the fact that alkaloidal therapy is founded upon the use of remedial agents in a state of chemic purity, completely prepared outside the body for ready ab. sorption, leaving practically no chemistry at all to be performed by the ailing and weakened cells.

The agents we utilize are the active principles of plants alkaloids, resinoids, glucosides and concentrations - and certain mineral preparations, all in a state of chemic purity. In fact, we use the same remedies we always did, but with the "dirt left out." For convenience in dispensing, these agents are usually given in the form of granules or minute tablets, accurately divided and measured to minimum dosage, to be given at frequent intervals until effect. By this means one is always absolutely safe, utilizing the smallest possible quantity of the best obtainable means to produce the desired therapeutic result. How to obtain this result most speedily, safely and pleasantly should be the study of every practician of our art. The use of these "arms of precision," in cumulative minimum doses, gives us such perfect control of the physiologic disturbance at the beginning of an acute disease that we promptly restore the normal equilibrium, jugulate the attack, and cure the patient before serious organic lesions have been produced. This effect may be secured in all cases depending upon a controllable congestion, such as bronchitis, pneumonia, peritonitis and kindred conditions. Eruptive fevers will pass through their cycle under any form of treatment, but when treated alkalometrically they do so in a mild and greatly modified form; while that class of affections of which typhoid fever may be taken as a type, may either be aborted in the early stages or, if seen too late for that, may be conducted through the regular course to a quick and safe termination, like the eruptive fevers. The early control of the congestive element means much as to the subsequent course of any febrile attack, whether it be that of a specific fever or of a local inflammation.

In the practice of alkaloidal therapy certain customs or basal principles have been developed, which, though not confined to this therapeutic method alone, have become, in a manner, habitual to its followers. Whether or not these principles are well-founded you may easily determine. I will first mention the giving of small doses, frequently repeated, until the desired effect is produced; that is, every fifteen to thirty minutes, according to the severity of the attack, until some improvement is manifest. Such doses should then be given, at greater intervals, as will keep up this effect. By adhering closely to this method it is simply impossible to overdose the patient. In this way the alkaloids and other active principles and the most powerful mineral drugs may be used with perfect safety. Here we have the first illustration of the value of the alkaloids in promoting accuracy of medication. These agents are clean-cut and well-defined in their effects and totally devoid of the uncertainty which hangs over the galenicals. For instance, when we give pilocarpin we direct the dose to be given every fifteen minutes until sweating occurs. We know this will be the effect; and no one who has enough intelligence to be left in charge of a patient can possibly mistake so simple a direction. But if we were to give the fluid extract of jarborandi we could not know whether it would induce perspiration or dry it up, because the way it will act depends on which of its alkaloids-pilocarpin or jaborin-happens to be in excess. Consequently we have to go into a long explanation to the nurse, which she is not likely to comprehend, because we do not really know just what effect the medicine is going to exert.

I will not occupy your time in detailing more of such illustrations, but will merely ask you to call to mind the great number of drugs that contain more than one active principle, some of which are antagonistic to the others, as in digitalis, ergot, hyoscyamus, opium, etc. Observations made in India upon the treatment of dysentery with ipecacuanha deprived of its emetin render it probable that many plants now thought to possess but one useful active principle may contain others of no less value. When applied 